\title{
Concurrent pathogenic variants in SLC6A1/ NOTCH1/PRIMPOL genes in a Chinese patient with myoclonic-atonic epilepsy, mild aortic valve stenosis and high myopia
}

Haiming Yuan ${ }^{1,2}$, Qingming Wang ${ }^{1,2}$, Yufeng Li $i^{1}$, Shuangxi Cheng ${ }^{1}$, Jianxin Liu ${ }^{1}$ and Yanhui Liu ${ }^{1,2^{*}}$

\begin{abstract}
Background: Pathogenic SLC6A1 variants have been reported in patients with myoclonic-atonic epilepsy (MAE). NOTCH1, encoding a member of the Notch family of proteins, is known to be associated with aortic valve disease. The PRIMPOL variant has only been identified in Chinese patients with high myopia. Exome sequencing analysis now allows the simultaneous detection of multiple genetic etiologies for patients with complicated clinical features. However, the presence of three Mendelian disorders in one patient supported by their respective pathogenic variants and clinical phenotypes is very rare.
\end{abstract}

Case presentation: Here, we report a 4-year-old Chinese boy who presented with MAE, delayed language, borderline intellectual disability (ID), mildly impaired social skills and attention deficit hyperactivity disorder (ADHD). He also had mild aortic valve stenosis and high myopia. Using whole-exome sequencing (WES), we identified three variants: (1) SLC6A1, NM_003042.4: c.881-883del (p.Phe294del), (2) NOTCH1, NM_017617.5:c.1100-2A > G and (3) PRIMPOL, NM_152683.4:c.265 T > G (p.Tyr89Asp). Parental Sanger sequencing confirmed that SLC6A1 and NOTCH1 variants were de novo, whereas the PRIMPOL variant was inherited from the father who also had high myopia. Furthermore, the PRIMPOL variant was absent from the genomes of the paternal grandparents, and thus was also a de novo event in the family. All three variants are classified as pathogenic.

Conclusion: The SLC6A1 variant could explain the features of MAE, delayed language, borderline ID, impaired social skills and ADHD in this patient, whereas the features of aortic valve stenosis and high myopia of the patient may be explained by variants in NOTCH1 and PRIMPOL, respectively. This case demonstrated the utility of exome sequencing in uncovering the multiple pathogenic variants in a patient with complicated phenotypes due to the blending of three Mendelian disorders.

Keywords: SLC6A1, NOTCH1, PRIMPOL, Myoclonic-atonic epilepsy, High myopia, Whole-exome sequencing

\footnotetext{
* Correspondence: yh523120@sina.com

'Dongguan Maternal and Child Health Care Hospital, Dongguan 523120,

China

${ }^{2}$ Dongguan Institute of Reproductive and Genetic Research, Dongguan

523120, China
}

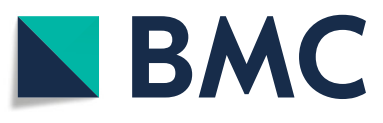

(c) The Author(s). 2020 Open Access This article is licensed under a Creative Commons Attribution 4.0 International License, which permits use, sharing, adaptation, distribution and reproduction in any medium or format, as long as you give appropriate credit to the original author(s) and the source, provide a link to the Creative Commons licence, and indicate if changes were made. The images or other third party material in this article are included in the article's Creative Commons licence, unless indicated otherwise in a credit line to the material. If material is not included in the article's Creative Commons licence and your intended use is not permitted by statutory regulation or exceeds the permitted use, you will need to obtain permission directly from the copyright holder. To view a copy of this licence, visit http://creativecommons.org/licenses/by/4.0/ The Creative Commons Public Domain Dedication waiver (http://creativecommons.org/publicdomain/zero/1.0/) applies to the data made available in this article, unless otherwise stated in a credit line to the data. 


\section{Background}

SLC6A1 (MIM 137165) encodes a gamma-aminobutyric acid (GABA) transporter known to be crucial for the reuptake of GABA from the synaptic cleft [1]. GABA is the main inhibitory neurotransmitter that counterbalances neuronal excitation in the brain, and disruption of this inhibitory balance can cause seizures. Previous reports have showed that pathogenic SLC6A1 variants are associated with MAE in patients by a loss-of-function mechanism in which SLC6A1 variants reduce or abolish the function of the GAT-1 GABA transporter [2-7]. In addition, patients with MAE may also present with variable degrees of intellectual disability, language impairment and behavioral problems, such as aggressive behavior/irritability, ADHD and autistic features [2, 4-6, 8].

NOTCH1 (MIM 190198) encodes a member of the Notch protein family, which includes NOTCH1, NOTCH2, NOTCH3 and NOTCH4 receptors [9]. Notch proteins belong to single-pass transmembrane receptors that regulate cell fate determinations during development, ensure crosstalk between different types of cells and their physiological differentiation, and are particularly critical in the development of cardiac valvulogenesis. Evidence suggests that pathogenic variants in this gene are related to aortic valve disease [10-14].

PRIMPOL (MIM 615421) encodes a primasepolymerase protein that is ubiquitous expressed in the eye and 26 other tissues [15]. Zhao et al. (2013) identified a heterozygous missense variant (c.265 T > G, p.Y89D) in PRIMPOL in affected members of a 4generation Chinese family with high myopia and in 4 sporadic Chinese patients with myopia. The PRIMPOL variant (p.Y89D) segregated with the disease and was absent in 270 Chinese controls, which implicated it in high myopia [16].

Here, we report three concurrent pathogenic variants in SLC6A1/NOTCH1/PRIMPOL genes in a Chinese boy with complicated clinical phenotypes, including MAE, delayed language, borderline ID, behavioral problems, aortic valve stenosis and high myopia.

\section{Case presentation}

The proband, a 4-year-old Chinese boy, was the firstborn to a nonconsanguineous couple and had a healthy younger brother. He was born at 38 weeks' gestation with a weight, height, and head circumference well within the normal ranges. His family history was unremarkable. He sat independently at 6 months, spoke his first word at 7 months, and walked without assistance at 13 months. At the age of 2 years 6 months, his language development showed a gradual delay. Seizures occurred at 2 years 8 months of age, characterized by initial cessation of activity and then progression to a fall sometimes followed by myoclonic movements. The event lasted for
$10 \mathrm{~s}$ each time with spontaneous remission. The patient was conscious throughout the seizures. Seizures were induced by fatigue, strong light and overexcitement. Seizure types observed included absences and myoclonicastatic, absence and partial seizures. Brain magnetic resonance imaging was normal at this age, but the electroencephalogram (EEG) was obviously abnormal due to paroxysms of bilateral independent and generalized 2.0$3.0 \mathrm{~Hz}$ frontally dominant high-voltage rhythmic spikes/ polyspikes-and-slow waves. After valproate therapy was initiated, his myoclonic seizures were controlled to an average of one episode per 2 weeks. He displayed borderline ID and mildly impaired language and had behavioral problems, including obvious ADHD, irritability, mildly impaired social reciprocity and poor eye contact.

Then, he was referred for a comprehensive clinical examination. Mild aortic valve stenosis was revealed by echocardiography. High myopia was also diagnosed, which was probably an inherited event because his father was also affected by high myopia during his early childhood without other suspected ocular diseases. However, his paternal grandparents had totally normal vision.

\section{Whole-exome sequencing (WES)}

This study was approved by the Committee on Ethics of the Dongguan Maternal and Child Health Care Hospital. DNA of family members was extracted from peripheral blood lymphocytes using standard methods and the sample from the proband was sent for whole exome sequencing. The parental DNA was used to confirm suspected variants by Sanger sequencing. WES was operated with an Illumina HiSeq 2500 system (Illumina). The bcl2fastq2 Conversion Software (v2.20) was employed for extracting Fastq files. BWA (v0.2.10) was used for genome alignments and variant detection. Clinic Sequence Analyzer (CSA) software was employed for biological analysis and interpretation. The pathogenicity of the sequence variants was interpreted in accordance with the American College of Medical Genetics and Genomics/Association for Molecular Pathology (ACMG/AMP) guidelines [17].

Using WES, a de novo inframe deletion variant at chr3:11067490-11,067,492 (hg19) in SLC6A1 was identified in our patient and was not observed in his parents or younger brother. The SLC6A1 change, c.881-883del (p.Phe294del), is predicted to cause the deletion of Phe (Fig. 1a). The Phe294 residue is highly conserved among different species. This inframe deletion variant is not present in either the 1000 genome databases or Exome Aggregation Consortium. Furthermore, this variant was previously reported to be a de novo event in patient 13, who was affected by seizures, described by Johannesen et al. (2018) [4]. Thus, this variant was categorized as clinically likely pathogenic according to ACMG/AMP 


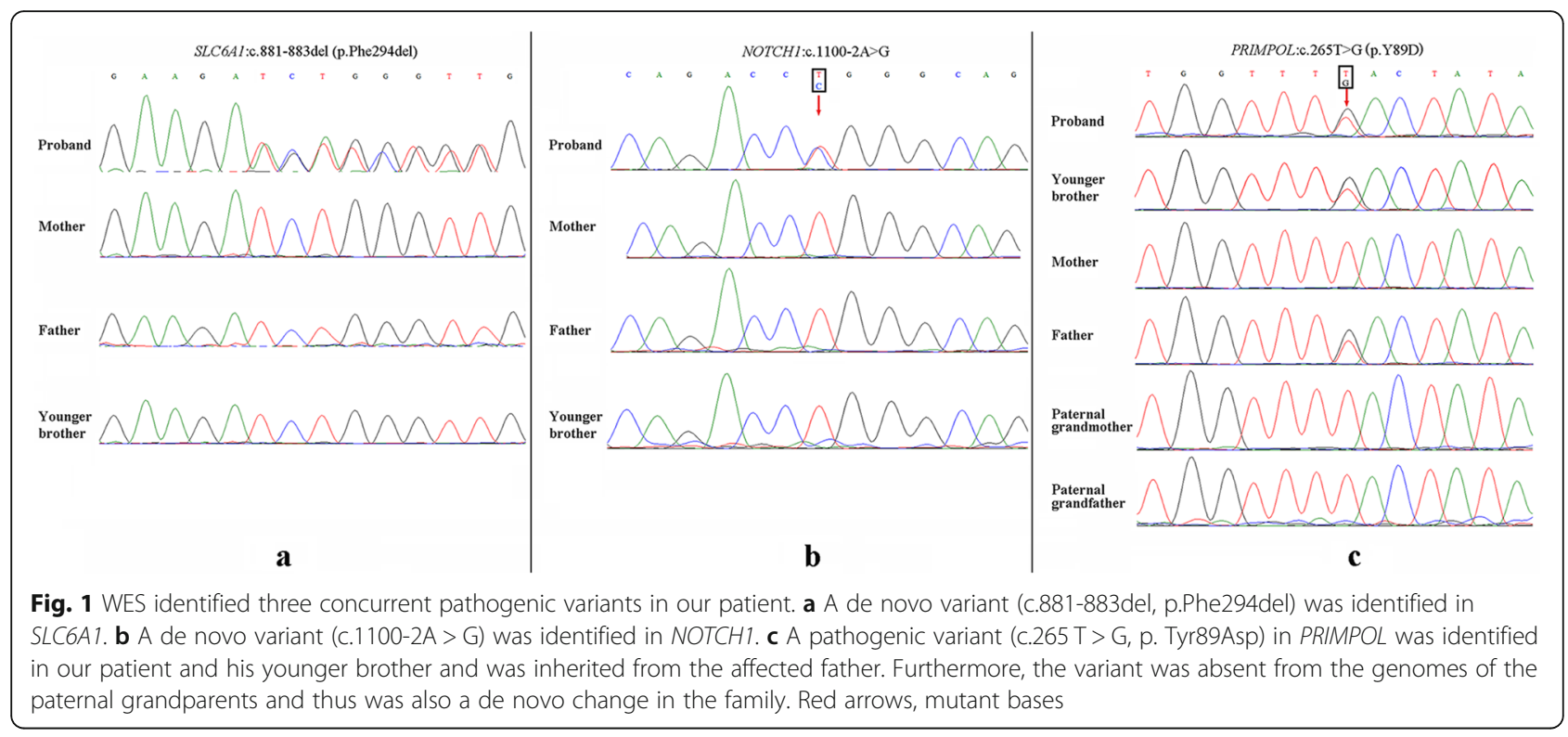

guidelines (PS2 + PM1 + PM2) (PS: pathogenic strong; PM: pathogenic moderate).

Then, the patient was given a comprehensive clinical examination. Unexpectedly, mild aortic valve stenosis and high myopia were revealed. Thus, WES data were reanalyzed to search for potential pathogenic variants causally linked with these clinical features. A de novo variant (c.1100-2A > G) in the NOTCH1 gene was identified in our patient, and his younger brother did not carry the variant (Fig. 1b). The variant alters the canonical splice acceptor site of intron 6. In silico analysis using Human Splicing Finder v3.0 (Aix Marseille University and Inserm, Marseille, France) predicted that this variant would eliminate the acceptor site, which was speculated to cause exon 7 to be incorrectly spliced out of the RNA transcript. Thus, this variant was classified as clinically pathogenic (PVS1 + PS2) (PVS1: pathogenic very strong). Finally, the variant (c.265 $\mathrm{T}>$ G, p.Tyr89Asp) in PRIMPOL was identified in the proband and his younger brother, who had not undergone an ophthalmic evaluation for his age, and was inherited from the affected father with high myopia. Further segregation analysis was performed and showed that the variant arose de novo in the father and was not seen in the paternal grandparents (Fig. 1c), moreover, the paternal grandparents had totally normal vision. Furthermore, functional studies have been established to support a damaging effect of the variant on PRIMPOL [18]. According to the ACMG/AMP guidelines, the variant was classified as pathogenic (PS2 + PS3 + PP1). The pedigree of our patient with three Mendelian disorders is depicted in Fig. 2.

\section{Discussion and conclusions}

Exome sequencing has been recommended as the first-tier clinical diagnostic test for individuals with neurodevelopmental disorders and provides opportunities for gaining insights into the relationships between specific multilocus genomic variations and disorders $[19,20]$. However, the presence of three Mendelian disorders in one patient supported by their respective pathogenic variants and clinical phenotypes is very rare.

In this study, we reported a 4-year-old Chinese boy who presented with MAE, borderline ID, delayed language, behavioral problems, mild aortic valve stenosis and high myopia. The three concurrent pathogenic variants were identified in our patient and were responsible for the complicated clinical phenotypes. Initially, the patient was referred to the clinic for MAE as the primary symptom at 2 years and 8 months of age. WES was performed for the patient and identified a de novo inframe deletion variant (c.881-883del, p.Phe294del) in SLC6A1 that has been reported in patients with MAE, but few SLC6A1 variant cases have been reported in Chinese patients. Previous reports indicated that individuals with MAE responded effectively to a ketogenic diet or valproic acid $[2,4,5]$. Thus, valproate therapy was recommended and greatly reduced his absence seizures to an average of one episode per 2 weeks. Individuals with MAE commonly display intellectual disability, language impairment and behavioral problems, including aggressive behavior/irritability, ADHD and autistic features [2, 4-6, 8]. Then, neurodevelopmental assessments were performed for the patient. As a result, he was diagnosed with borderline ID, mild delayed language, and behavioral problems, including obvious ADHD, irritability, mild impaired social reciprocity and poor eye contact. The severity of the clinical presentation may differ among patients carrying SLC6A1 variants, even between patients with the same mutation [2, 4]. Our patient 


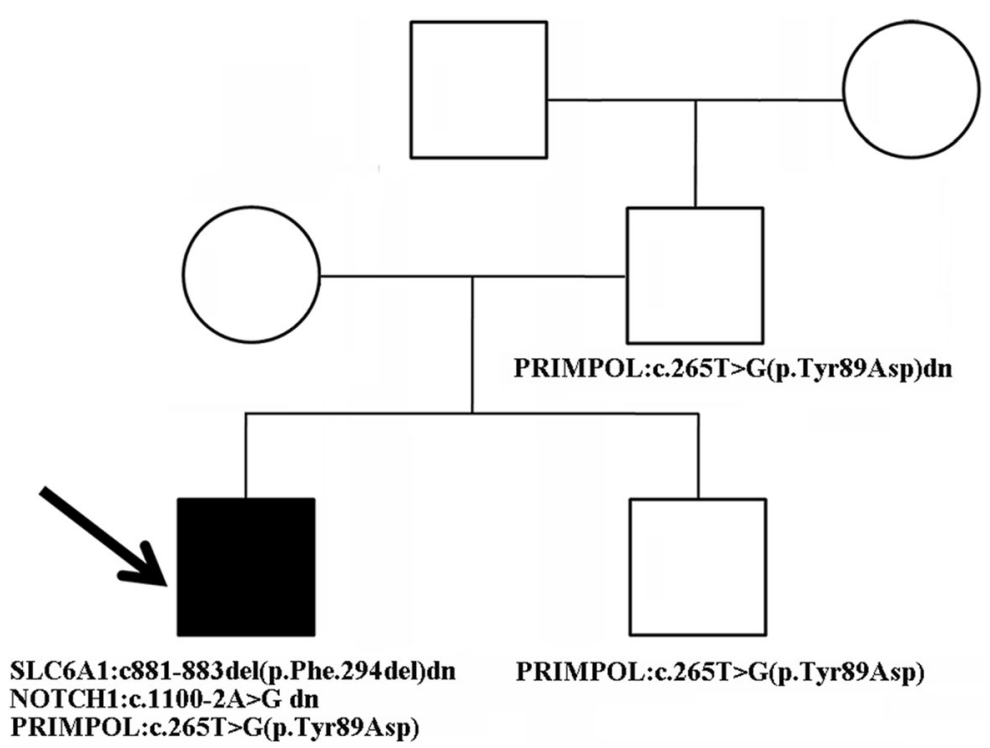

Fig. 2 Pedigree of our proband with the variants in SLC6A1, NOTCH1 and PRIMPOL. The two variants in SLC6A1 and NOTCH1 were de novo events, and the PRIMPOL variant was inherited from the affected father, which was also a de novo change since the variant was absent from the paternal grandparents. Proband is marked with an arrow. dn: de novo

carried the same mutation as patient 13 (Johannesen et al., 2018) [4]. Our patient displayed borderline ID, whereas patient 13 had mild ID before epilepsy onset and moderate ID after epilepsy. Our patient had irritability, whereas patient 13 did not show this feature, but both presented with ADHD. Patient 13 had mild ataxia, whereas our patient did not. Patient 13 became seizurefree with the combined treatment of lamotrigine and ethosuximide, whereas our patient nearly became seizure-free with valproic acid being the most effective drug. Thus, both the severity and prognosis of our patient's clinical manifestations were better than those of the patient 13. However, cognitive competence and language development should be persistently followed for our patient.

Mild aortic valve stenosis and high myopia as incidental clinical features were revealed for our patient through a comprehensive clinical exam. Then, WES data reanalysis was initiated to search for probable pathogenic variants causally linked with these clinical phenotypes. In return, a de novo variant (c.1100-2A > G) in NOTCH1 and a pathogenic variant (c.265 T > G, p.Tyr89Asp) in PRIMPOL inherited from his father with high myopia were identified, which were missed in the initial data analysis. The main reasons for the initial negative result were due to incomplete recognition of the patient's phenotypes. Therefore, the improvement in access to more detailed phenotypic data of the patients and the possibility of estimating the changing phenotype over time would contribute to the increased diagnostic yield [21].
Echocardiography revealed mild aortic valve stenosis, which may be a consequence of the de novo variant (c.1100-2A > G) in NOTCH1 [10-14]. At present, mild aortic valve stenosis does not have an impact on his daily life, but it needs to be regularly examined.

The variant (c.265 T $>$ G, p.Tyr89Asp) in PRIMPOL was originally identified in affected members of a 4generation Chinese family with high myopia and in 4 sporadic Chinese patients with myopia. The evidence that the variant segregated with disease and was absent in 270 Chinese controls indicated that the variant (p.Y89D) in PRIMPOL was associated with high myopia [16]. Furthermore, Keen et al. (2014) performed a functional study for the variant, unequivocally establishing that this variant has a significant impact on the function of this enzyme [18]. However, Li and Zhang (2015) provided contradictory evidence that the variant was identified in two of 407 patients with high myopia, 13 of 813 patients with other ocular diseases, and seven of 384 normal controls [22]. Thus, further work is required to establish the relationship between PRIMPOL and high myopia [22, 23]. There is no doubt that myopia is a complex genetic trait. However, our patient was only a 4-year-old boy with high myopia, who was obviously little affected by environmental factors. Furthermore, WES also excluded other known genetic causes responsible for ocular disorders to the greatest degree possible. Next, we performed further segregation analysis in the members of the family and showed that the variant was inherited from the father who had been affected by high myopia since early childhood without other suspected 
ocular diseases, which was a de novo event in that it was not present in the paternal grandparents who had totally normal vision. The evidence for cosegregation in our family may support a role for PRIMPOL in high myopia.

WES has allowed clinicians to better understand the pathogenesis of patients with complicated clinical features [19-21]. The improvement of clinical phenotypes is very important for the interpretation or reanalysis of WES data, and the incidental findings of WES analysis will also promote the revelation of relevant phenotypes, as both complement each other and ultimately form a closed loop for accurate diagnosis of genetic diseases.

\section{Abbreviations}

MAE: Myoclonic-atonic epilepsy; ID: Intellectual disability; ADHD: Attention deficit hyperactivity disorder; WES: Whole-exome sequencing;

GABA: Gamma-aminobutyric acid

\section{Acknowledgments}

We would like to express our sincere gratitude to our patient and his family for their cooperation.

\section{Authors' contributions}

HMY drafted the first versions of the manuscript. HMY and YHL were responsible for the design of the project, data analysis, and manuscript writing. QMW, YFL, SXC and JXL assisted in the literature review and participated in the experiments, data entry, and data analysis phases of the project. All authors have read and approved the final version of the manuscript.

\section{Funding}

This study was supported by the Dongguan Social Development Project (No. 201950715007158 to Yanhui Liu). The funding body participated in the design of the project and interpretation of whole-exome sequencing data.

\section{Availability of data and materials}

The data used and/or analyzed in the present report was deposited in the NCBI BioProject database. The data is accessible via the accession number: PRJNA627312; or via the links: https://www.ncbi.nlm.nih.gov/bioproject/ PRJNA627312

\section{Ethics approval and consent to participate}

All procedures in this study were approved by the Committee on Ethics of the Dongguan Maternal and Child Health Care Hospital. Detailed written informed consent was obtained from all participants. In the case of children or those cognitively impaired and unable, written informed consent for participation in this study was obtained from the patients' parents or guardians.

\section{Consent for publication}

Written informed consent for publication of clinical and genetic data was obtained from all participants. The parents /legal guardian of the children (under the age of 18) has signed written informed consent for publication of clinical and genetic data.

\section{Competing interests}

The authors declare that they have no competing interests.

Received: 8 April 2020 Accepted: 27 April 2020

Published online: 06 May 2020

\section{References}

1. Durkin MM, Smith KE, Borden LA, et al. Localization of messenger RNAs encoding three GABA transporters in rat brain: an in situ hybridization study. Brain Res Mol Brain Res. 1995;33:7-21.

2. Carvill GL, McMahon JM, Schneider A, et al. Mutations in the GABA transporter SLC6A1 cause epilepsy with myoclonic-atonic seizures. Am J Hum Genet. 2015;96:808-15.
3. Halvorsen M, Petrovski S, Shellhaas R, et al. Mosaic mutations in early-onset genetic diseases. Genet Med. 2016;18:746-9.

4. Johannesen KM, Gardella E, Linnankivi T, et al. Defining the phenotypic spectrum of SLC6A1 mutations. Epilepsia. 2018:59:389-402.

5. Palmer S, Towne MC, Pearl PL, et al. SLC6A1 mutation and ketogenic diet in epilepsy with myoclonic-atonic seizures. Pediatr Neurol. 2016;64:77-9.

6. Zech $M$, Jech $\mathrm{R}$, Wagner $\mathrm{M}$, et al. Molecular diversity of combined and complex dystonia: insights from diagnostic exome sequencing. Neurogenetics. 2017;18:195-205.

7. Mattison KA, Butler KM, Inglis GAS, Dayan O, Boussidan H, Bhambhani V, Philbrook B, da Silva C, Alexander JJ, Kanner BI, Escayg A. SLC6A1 variants identified in epilepsy patients reduce $\gamma$-aminobutyric acid transport. Epilepsia. 2018;59(9):e135-41.

8. Yuan FF, Gu X, Huang X, Zhong Y, Wu J. SLC6A1 gene involvement in susceptibility to attention-deficit hyperactivity disorder. Prog NeuroPsychopharmacol Biol Psychiatry. 2017;77:202-8.

9. Das I, Craig C, Funahashi Y, Jung KM, Kim TW, Byers R, et al. Notch oncoproteins depend on gamma-secretase/presenilin activity for processing and function. J Biol Chem. 2004;279(29):30771-80.

10. Garg V, Muth AN, Ransom JF, Schluterman MK, Barnes R, King IN, et al. Mutations in NOTCH1 cause aortic valve disease. Nature. 2005;437:270-4.

11. Kerstjens-Frederikse WS, van de Laar IM, Vos YJ, Verhagen JM, Berger RM, Lichtenbelt KD, et al. Cardiovascular malformations caused by NOTCH1 mutations do not keep left: data on 428 probands with left-sided CHD and their families. Genet Med. 2016;18(9):914-23.

12. Mohamed SA, Aherrahrou Z, Liptau H, Erasmi AW, Hagemann C, Wrobel S, et al. Novel missense mutations (p.T596M and p.P1797H) in NOTCH1 in patients with bicuspid aortic valve. Biochem Biophys Res Commun. 2006; 345(4):1460-5

13. Kostina AS, Uspensky VE, Irtyuga OB, Ignatieva EV, Freylikhman O, Gavriliuk ND, et al. Notch-dependent EMT is attenuated in patients with aortic aneurysm and bicuspid aortic valve. Biochim Biophys Acta. 2016;1862(4):733-40.

14. McBride KL, Riley MF, Zender GA, Fitzgerald-Butt SM, Towbin JA, Belmont JW, Cole SE. NOTCH1 mutations in individuals with left ventricular outflow tract malformations reduce ligand-induced signaling. Hum Mol Genet. 2008; 17(18):2886-93.

15. Fagerberg L, Hallström BM, Oksvold P, Kampf C, Djureinovic D, Odeberg J et al. Analysis of the human tissue-specific expression by genome-wide integration of transcriptomics and antibody-based proteomics. Mol Cell Proteomics. 2014;13(2):397-406.

16. Zhao F, Wu J, Xue A, Su Y, Wang X, Lu X, et al. Exome sequencing reveals CCDC111 mutation associated with high myopia. Hum Genet. 2013;132:913-21.

17. Richards S, Aziz N, Bale S, Bick D, Das S, Gastier-Foster J, et al. Standards and guidelines for the interpretation of sequence variants: a joint consensus recommendation of the American College of Medical Genetics and Genomics and the Association for Molecular Pathology. Genet Med. 2015;17(5):405-24.

18. Keen BA, Bailey LJ, Jozwiakowski SK, Doherty AJ. Human PrimPol mutation associated with high myopia has a DNA replication defect. Nucleic Acids Res. 2014;42:12102-11.

19. Srivastava S, Love-Nichols JA, Dies KA, Ledbetter DH, Martin CL, Chung WK, Firth HV, Frazier T, Hansen RL, Prock L, Brunner H, Hoang N, Scherer SW, Sahin M, Miller DT, NDD Exome Scoping Review Work Group. Meta-analysis and multidisciplinary consensus statement: exome sequencing is a first-tier clinical diagnostic test for individuals with neurodevelopmental disorders. Genet Med. 2019. https://doi.org/10.1038/s41436-019-0554-6.

20. Posey JE, Harel T, Liu P, Rosenfeld JA, James RA, Coban Akdemir ZH, et al. Resolution of disease phenotypes resulting from multilocus genomic variation. N Engl J Med. 2017;376(1):21-31.

21. Salmon LB, Orenstein N, Markus-Bustani K, Ruhrman-Shahar N, Kilim Y, Magal $N$, et al. Improved diagnostics by exome sequencing following raw data reevaluation by clinical geneticists involved in the medical care of the individuals tested. Genet Med. 2019;21(6):1443-51.

22. Li J, Zhang Q. PRIMPOL mutation: functional study does not always reveal the truth. Invest Ophthalmol Vis Sci. 2015;56(2):1181-2.

23. Keen BA, Bailey LJ, Jozwiakowski SK, Doherty AJ. Author response: PRIMPOL mutation: functional study does not always reveal the truth. Invest Ophthalmol Vis Sci. 2015;56(2):1183.

\section{Publisher's Note}

Springer Nature remains neutral with regard to jurisdictional claims in published maps and institutional affiliations. 